\title{
Recovery in Supported Accommodations: A Scoping Review and Synthesis of Interventions for People with Severe Mental Illness
}

\author{
Neis Bitter ${ }^{1}$ Diana Roeg ${ }^{1}\left[\right.$ (D) Chijs van Nieuwenhuizen ${ }^{1,2} \cdot$ Jaap van Weeghel ${ }^{1,3,4}$
}

Received: 26 July 2019 / Accepted: 26 January 2020 / Published online: 3 February 2020

(c) The Author(s) 2020

\begin{abstract}
Research on the recovery domains beside clinical recovery of people with severe mental illness in need of supported accommodations is limited. The aim of this study was (1) to investigate which recovery interventions exist for this group of people and (2) to explore the scientific evidence. We conducted a scoping review, including studies with different designs, evaluating the effectiveness the recovery interventions available. The search resulted in 53 eligible articles of which 22 focused on societal recovery, six on personal recovery, five on functional recovery, 13 on lifestyle-interventions, and seven on creative and spiritual interventions. About a quarter of these interventions showed added value and half of them initial promising results. The research in this area is still limited, but a number of recovery promoting interventions on other areas than clinical recovery have been developed and evaluated. Further innovation and research to strengthen and repeat the evidence are needed.
\end{abstract}

Keywords Mental health recovery $\cdot$ Societal participation $\cdot$ Severe mental illness $\cdot$ Supported accommodation $\cdot$ Supported housing

\section{Introduction}

Most people with severe mental health problems can recover and live in the community with or without support (Keet et al. 2019). A relatively small group of people (10-20\%) has long-term, severe and complex needs but consumes $25-50 \%$ of the mental health and social care budget (Killaspy et al.

Diana Roeg

d.p.k.roeg@tilburguniversity.edu

Neis Bitter

n.bitter@rivierduinen.nl

Chijs van Nieuwenhuizen

ch.vannieuwenhuizen@tilburguniversity.edu

Jaap van Weeghel

j.vanweeghel@tilburguniversity.edu

1 Department of Social and Behavioural Sciences, Tranzo Scientific Center for Care and Wellbeing, Tilburg University, PO Box 90153, 5000, LE, Tilburg, The Netherlands

2 GGzE Institute for Mental Health Care, PO Box 909, 5600, AX, Eindhoven, The Netherlands

3 Phrenos Centre of Expertise, PO Box 1203, 3500, BE, Utrecht, The Netherlands

4 Parnassia Group, Dijk en Duin Mental Health Centre, PO Box 305, 1900, AH, Castricum, The Netherlands
2016). Killaspy et al. (2016) therefore referred to this group as a 'low volume, high needs' group. These people often have major negative and ongoing positive symptoms in addition to other mental, social and physical health problems. They need the permanent support of supported housing facilities or residential care (Killaspy 2016; Leff et al. 2015; Sandhu et al. 2017; van Hoof et al. 2015). These services offer practical daily care, nursing and support to persons with severe mental illness (SMI) in their daily lives, aiming at improvements in recovery and functioning. Nevertheless, people with long-term SMI still report unmet needs concerning health, work, social relations and daily activities (Bitter et al. 2016; de Heer-Wunderink et al. 2012a, b).

Over the past two decades, there have been increasing attention for what it means to recover from a mental illness. There is a growing recognition that recovery is more than the remission of psychiatric symptoms. The current vision is that recovery is 'a way of living a satisfying, hopeful and contributing life even with limitations caused by illness' (Anthony 1993). Several authors described that recovery comprises multiple aspects (Couwenbergh and van Weeghel 2014; Davidson et al. 2005; Leamy et al. 2011; Resnick et al. 2005). An example of a classification that is used often in the Netherlands is: clinical, functional, social and personal recovery (Couwenbergh and van Weeghel 2014). First, 
clinical recovery refers to a decrease in clinical symptoms such as hallucinations, anxiety or depressive feelings (Liberman et al. 2002). The other dimensions are of more recent attention. Functional recovery refers to executive functioning such as planning and problem solving (Savla et al. 2012). Societal recovery is about regaining everyday functioning in areas such as work, social relationships, housing and leisure (Farkas and Anthony 2010). Personal recovery refers to a person's own experience and is about hope, empowerment, self-determination and regaining the identity of someone who is living a meaningful life despite the presence of symptoms (Anthony 1993; van Gestel-Timmermans et al. 2012a). Recovery dimensions are closely related and influence each other constantly in complex processes (Davidson et al. 2005).

Treatment and support for people with SMI therefore should ideally focus on all dimensions of recovery and be tailored to a person's individual needs (Bitter et al. 2016; van Weeghel et al. 2019a). Several types of psychosocial interventions have been developed to support people with SMI in their recovery on the dimensions next to the clinical one (Slade et al. 2014). Rehabilitation methods, for example, focus on clients' personal goals and wishes regarding daily life and societal recovery. Examples of well-known methods in this field are the 'choose-get-keep' approach, also referred to as Boston psychiatric rehabilitation, (Anthony et al. 2002), illness management and recovery (IMR) (Mueser et al. 2006) and the strengths model of case management (Rapp and Goscha 2006). Other methods focus on a specific aspects of life. These include individual placement and support (IPS) in which people are supported to gain and stay in competitive employment (Burns et al. 2007; Michon et al. 2011). Other methods aim to improve cognitive functioning or practical skills; these include social and independent living skill modules, cognitive remediation programs and cognitive adaptation training (CAT) (Hansen et al. 2012; Marder et al. 1996; Stiekema et al. 2015). More recently, interventions have been developed especially focusing on personal recovery, sometimes provided by experts-byexperience (Boevink et al. 2016; Fox and Horan 2016; van Gestel-Timmermans et al. 2012b).

There is an increasing amount of research on the effectiveness of interventions addressing several outcomes. IPS, for example, has shown to have a strong and consistent effect on vocational outcomes (Michon et al. 2011). Furthermore, the Boston approach has been shown to increase social functioning and goal attainment (Swildens et al. 2011). Studies concerning several other interventions, such as the strengths model and those aimed at personal recovery, have reported varying results (Ibrahim et al. 2014; Lloyd-Evans et al. 2014; Tse et al. 2016).

Although research on these interventions have shown promising results, studies on interventions for clients living in supported accommodations such as residential care and supported housing services, however, lack behind (Chilvers et al. 2006; McPherson et al. 2018). Available studies were executed mainly with participants who live independently with a relative small amount of support. Also, most of the available studies concern interventions that focus on a selective group of motivated clients who can formulate concrete goals (Michon et al. 2011; Swildens et al. 2011). We cannot assume that these practices are suitable and valuable for people with SMI living in supported accommodations, of which is known their needs are more complex and some have lost their motivation and goals in life (Bitter et al. 2016; de Heer-Wunderink 2012).

For that reason, this study aims to identify and evaluate studies on psychosocial interventions focusing on the dimensions of recovery besides the clinical one, in supported accommodation for people with severe mental illness. The findings of this study can contribute to the further development of the content and quality of the support offered by supported accommodation.

\section{Aims of the Study}

With this review, we aim to answer the following questions:

1. Which interventions have been applied and evaluated to support clients with severe mental illness using supported accommodation in their recovery on domains besides clinical recovery?

2. What scientific evidence is available about the outcomes of these interventions?

\section{Methods}

We choose to conduct a scoping review, as these are established for use when the objective is to examine the extent, range and nature of research activity in a certain field and to summarize and disseminate the research findings (Pham et al. 2014). We followed the steps described by Arksey and O'Malley (2005) in their framework for the execution of a scoping review: (a) identify the research question, (b) identify relevant studies, (c) select the studies, (d) chart the data and (e) collate, summarize and report the results.

\section{Search Strategy}

To answer our first research question, we searched the following databases: PubMed, Psycinfo, Embase and Cinahl (January 2018, Update December 2019). These databases were chosen to cover medical (PubMed and Embase) as well as psychological (Psycinfo) and nursing (Cinahl) literature. We formulated and combined search terms concerning: (a) 
the setting and population (mental disorder/illness, schizophrenia, psychosis, inpatient rehabilitation, supported accommodation, sheltered housing, housing facility, community housing, community facility, supported housing, residential facility and residential care), (b) the scope and outcome of the intervention (psychosocial, societal, recovery, functioning, rehabilitation, health, wellness and cognition), and (c) study type (clinical trial, randomized controlled trial, evaluation study, experimental trial, naturalistic study, follow up study, quasi-experimental and case study).

To select studies that corresponded with our research aims, we formulated inclusion and exclusion criteria. We included peer-reviewed articles that were published in English from January 2000 till December 2019; aimed at adult clients with severe mental illness receiving services from housing services or comparable long-term ( $>1$ year) supported accommodation; evaluated psychosocial interventions focussing on personal, functional or societal recovery outcomes; evaluated the outcomes of an intervention on the client level; and evaluated outcomes by means of effect evaluation all types of designs except for expert opinions and case studies. As we aimed to give an overview of existing interventions for this group, we also included protocol papers and checked if there were results published already. To be able to provide a clearly defined answer to the research questions and to keep the results manageable, we also formulated exclusion criteria. Studies were excluded if they primarily focussed on substance abuse; intellectual and/or developmental disability, including brain damage; or on homelessness; or if they were executed in developing countries.

\section{Study Selection Process}

In the first and second selection phase, the first two authors each screened a separate part of the titles from the initial search, and of the remaining papers they screened the abstracts on relevance. When there was doubt, the selection was made in consensus. The first and second author determined final inclusion by discussing the interpretation of the inclusion criteria in certain cases. When doubt persisted about an abstract, the article was included so that a more careful decision could be made in the next phase.

In the third phase, the first and second author read the full-text of the remaining articles and made a final selection. In this final phase, both authors each read half of the articles independently. Again, articles about which doubt existed were discussed until consensus was reached. The selected studies then were categorised in a qualitative synthesis, based on the dimensions of recovery: societal, functional and personal, and additional in vivo categories were made when needed.

\section{Outcome Evaluation}

Our second aim was to evaluate what is already known about the outcomes of these interventions. Therefore, the second phase of the qualitative synthesis was evaluation of each study to understand the status of the available evidence of each intervention found. First, we formulated categories of designs based on Evans' hierarchy of evidence (2003): randomized (controlled) study, uncontrolled longitudinal study, or other (all other designs except case studies and expert opinions). Next, we evaluated the results of relevant outcomes and (where possible) the effect sizes of these results. Again, three options were possible: Large or medium effects, small effects, or neutral, unclear, unknown or not convincing yet. Based on these criteria, we concluded there was one of three options: (a) added value when a randomized control trial (RCT) resulted in small, large or medium effects, (b) promising first results when other designs than RCTs showed positive results, or (c) no evidence for the effectiveness yet when there were neutral or negative results or no results yet. The first and second author executed this quality assessment independently. Each assessed an equal part and then discussed the results until they reached a consensus. This review is part of a larger research project which received ethical approval from the Medical Research Ethics Committee of the Elisabeth Hospital in Tilburg (NL41169.008.12).

\section{Results}

Fifty three articles met the inclusion criteria. Figure 1 shows the PRISMA flow diagram of the search, while Table 1 shows the results of the qualitative synthesis of the included articles. Five categories were formed. Three were based on the often distinguished dimensions of the recovery process: societal recovery, personal recovery and functional recovery, and two were formed in vivo: lifestyle, and cultural and spiritual.

Most of the included studies focused on societal recovery $(n=22)$, addressing psychiatric rehabilitation approaches, occupational therapy and skills training. Studies concerned with personal recovery $(n=6)$ focused on peer-run programs, illness management and recovery, and interventions aiming at increasing empowerment. Studies in the functional recovery category $(n=5)$ examined cognitive training or remediation. Those in the lifestyle category $(n=13)$ were aimed at a healthy lifestyle, (e.g. physical exercise and healthy eating). The last category, cultural and spiritual interventions $(n=7)$, looked at tai chi, music therapy and art therapy. 
Fig. 1 PRISMA flow diagram

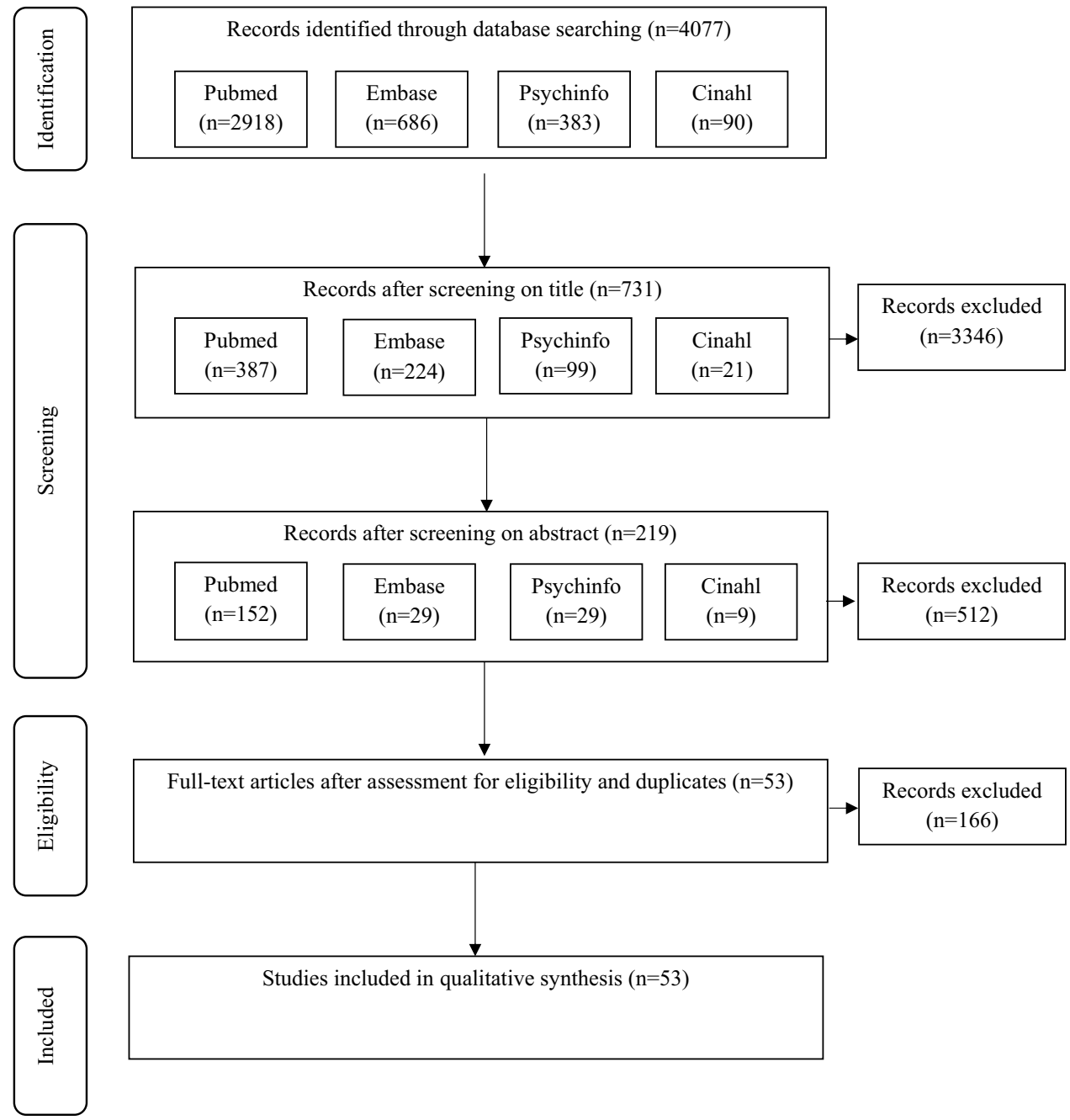

Table 1 Type, amount and evidence of included studies

\begin{tabular}{|c|c|c|c|}
\hline Type of intervention & Including & No. of studies & Evidence \\
\hline Societal recovery & $\begin{array}{l}\text { Approaches aiming at personal goals, (social) skills training, } \\
\text { occupational therapy }\end{array}$ & 22 & $\begin{array}{l}4 \text { added value } \\
11 \text { promising results } \\
7 \text { no evidence yet }\end{array}$ \\
\hline Personal recovery & Peer run, empowerment, confidence, hope, meaning & 6 & $\begin{array}{l}2 \text { added value } \\
4 \text { promising results }\end{array}$ \\
\hline Functional recovery & Cognitive remediation/training, cognitive adaptation & 5 & $\begin{array}{l}3 \text { added value } \\
2 \text { no evidence yet }\end{array}$ \\
\hline Lifestyle & Health promotion, exercise, healthy meals & 13 & $\begin{array}{l}7 \text { promising results } \\
6 \text { no evidence yet }\end{array}$ \\
\hline Spiritual and creative & Tai chi, music therapy, art therapy & 7 & $\begin{array}{l}3 \text { added value } \\
3 \text { promising results } \\
1 \text { no evidence yet }\end{array}$ \\
\hline
\end{tabular}

\section{Evaluation of Results of the Interventions}

We evaluated the outcomes of all included studies (see Table 2 for a summary). Following is a description of the overall picture for each category.

\section{Societal Recovery}

This category contains the greatest number of studies $(n=22)$. These studies focussed on diverse interventions. Nine evaluated interventions aimed at general goal 


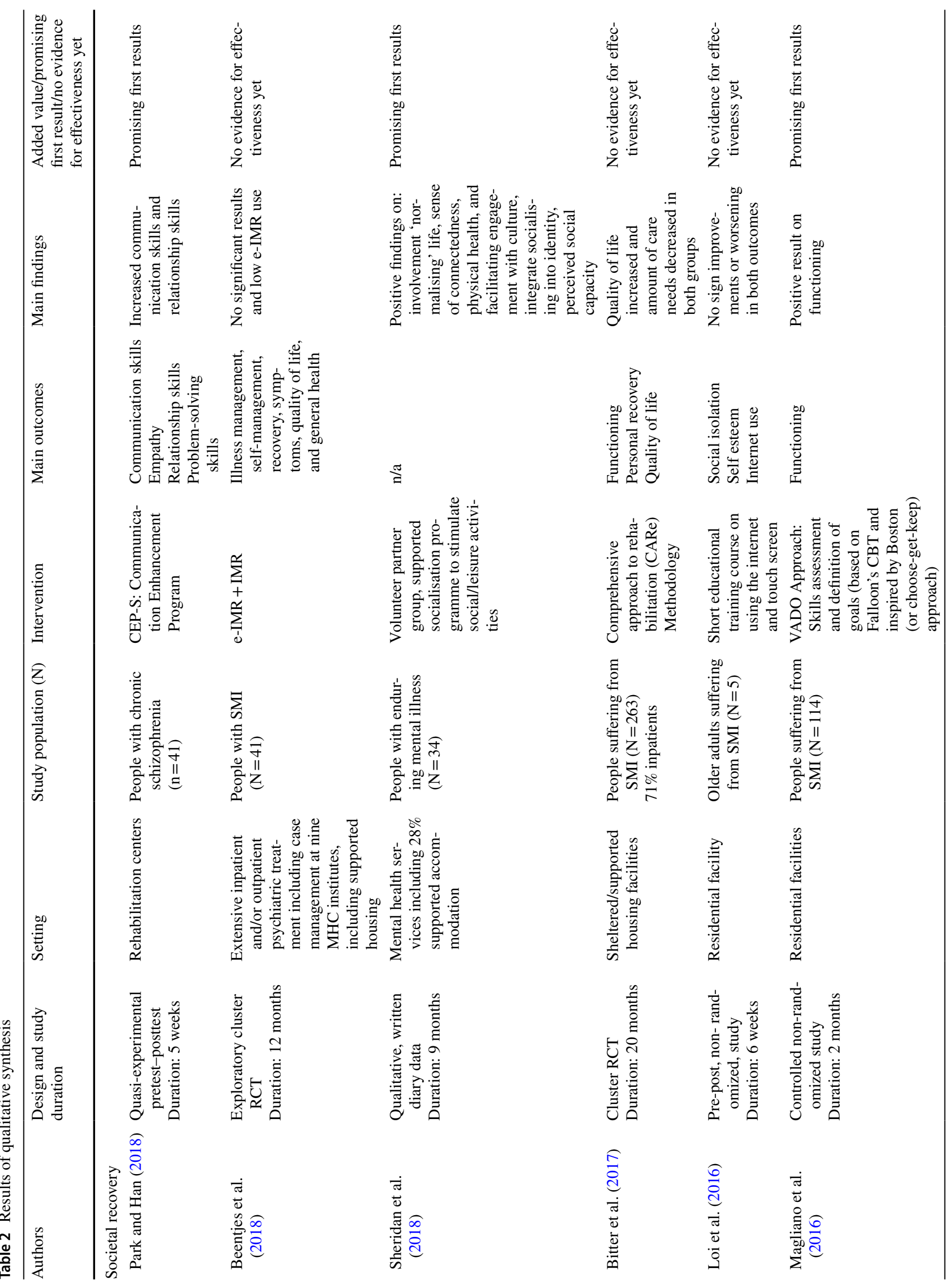




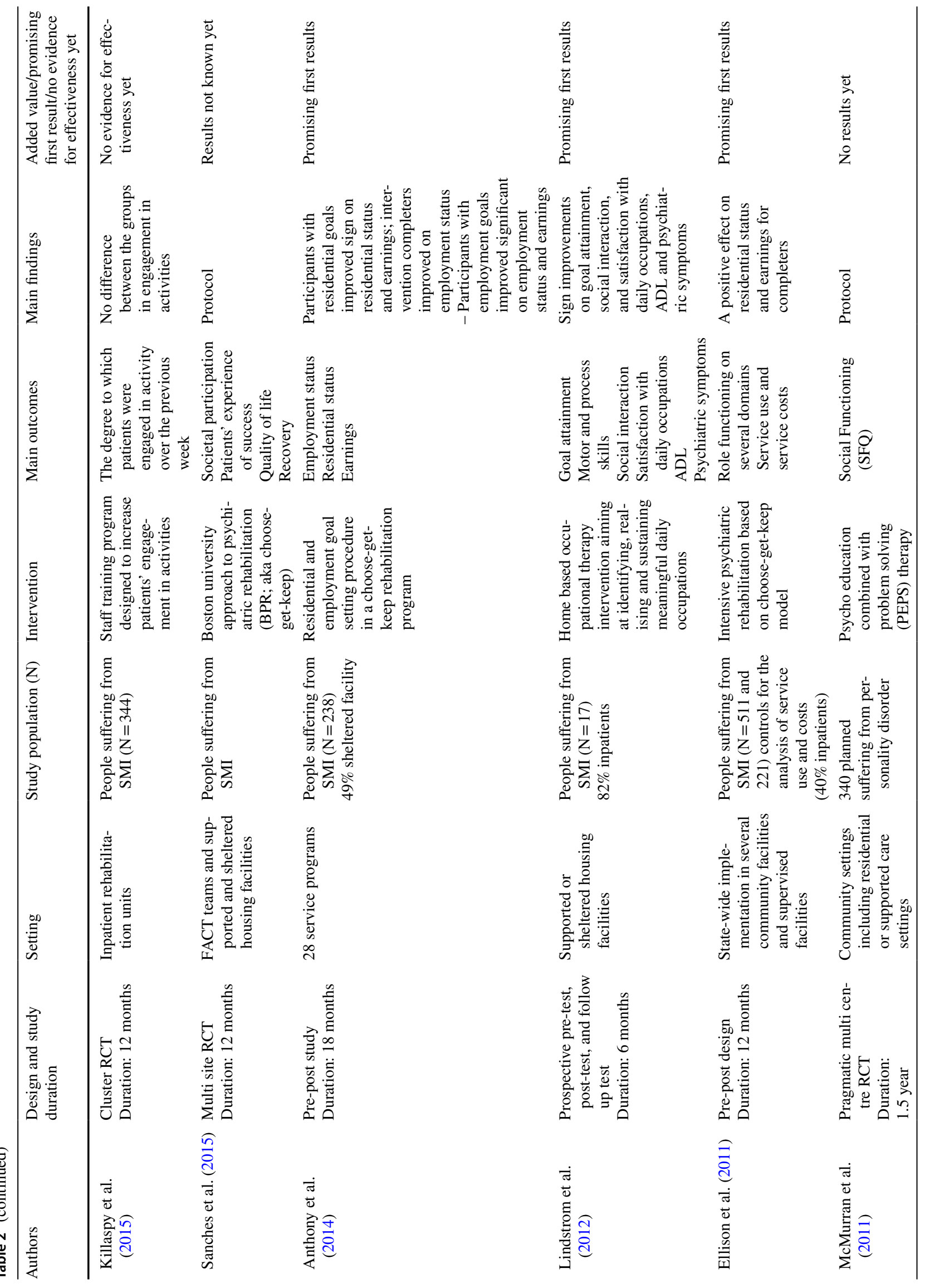




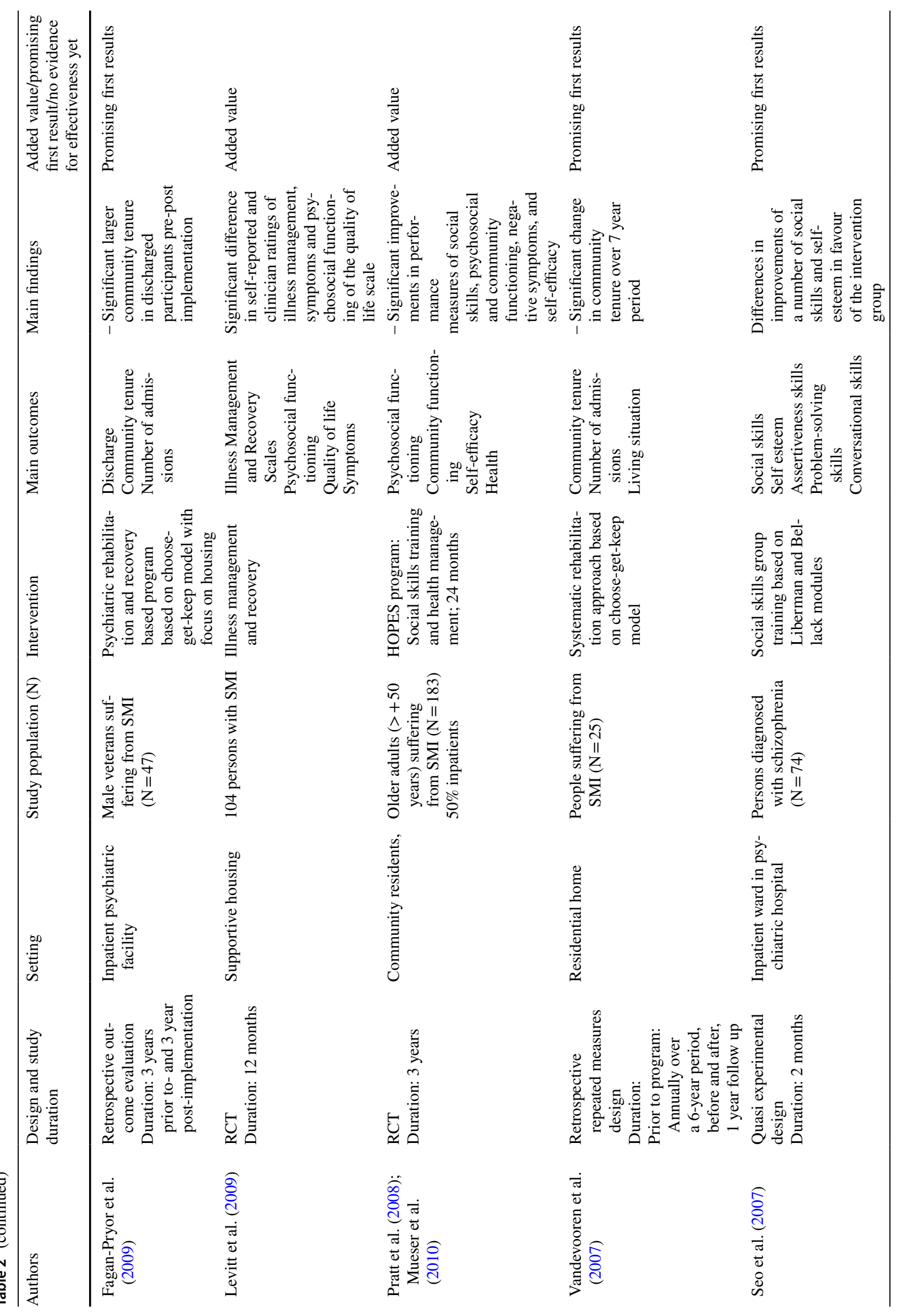




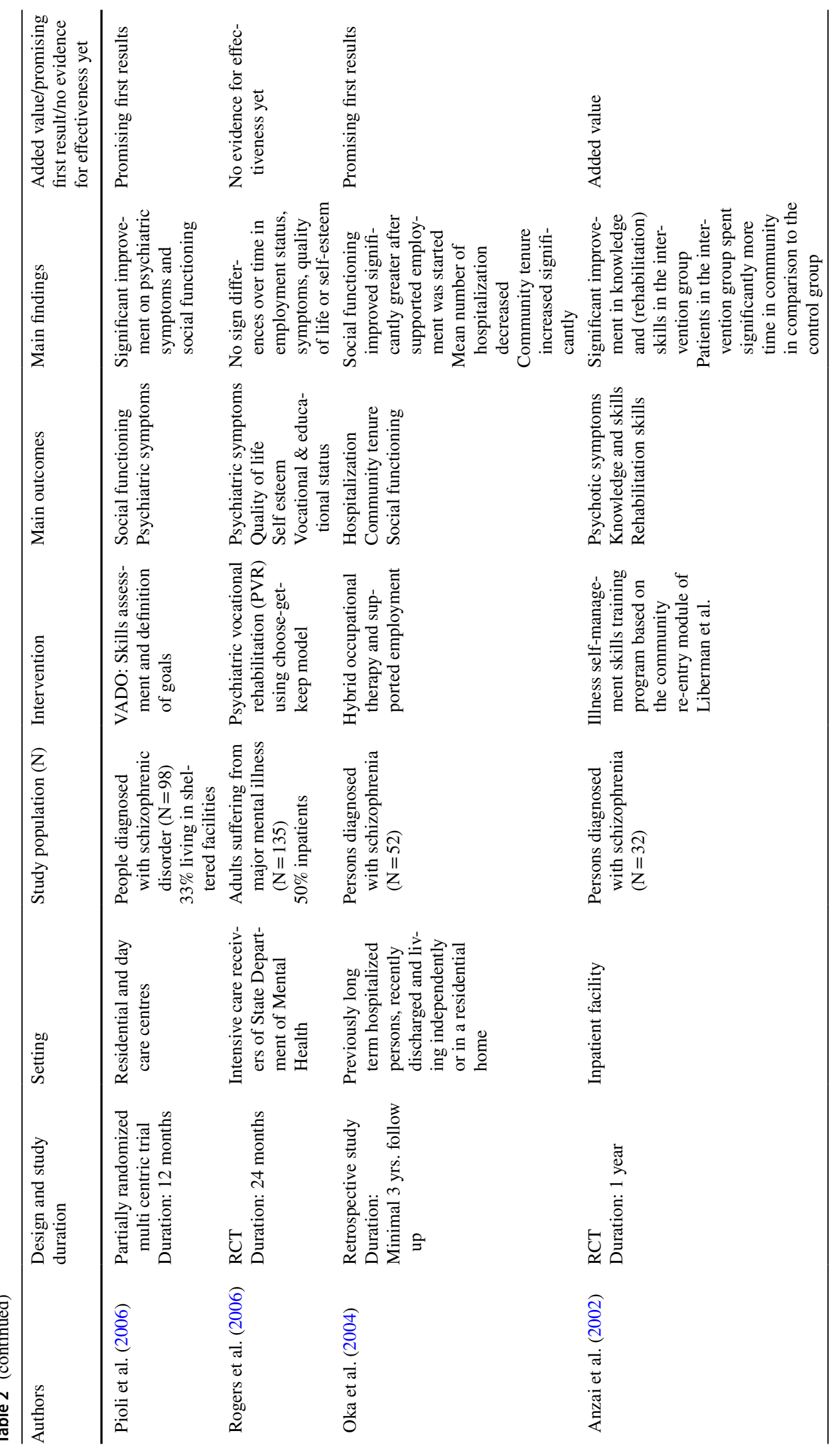




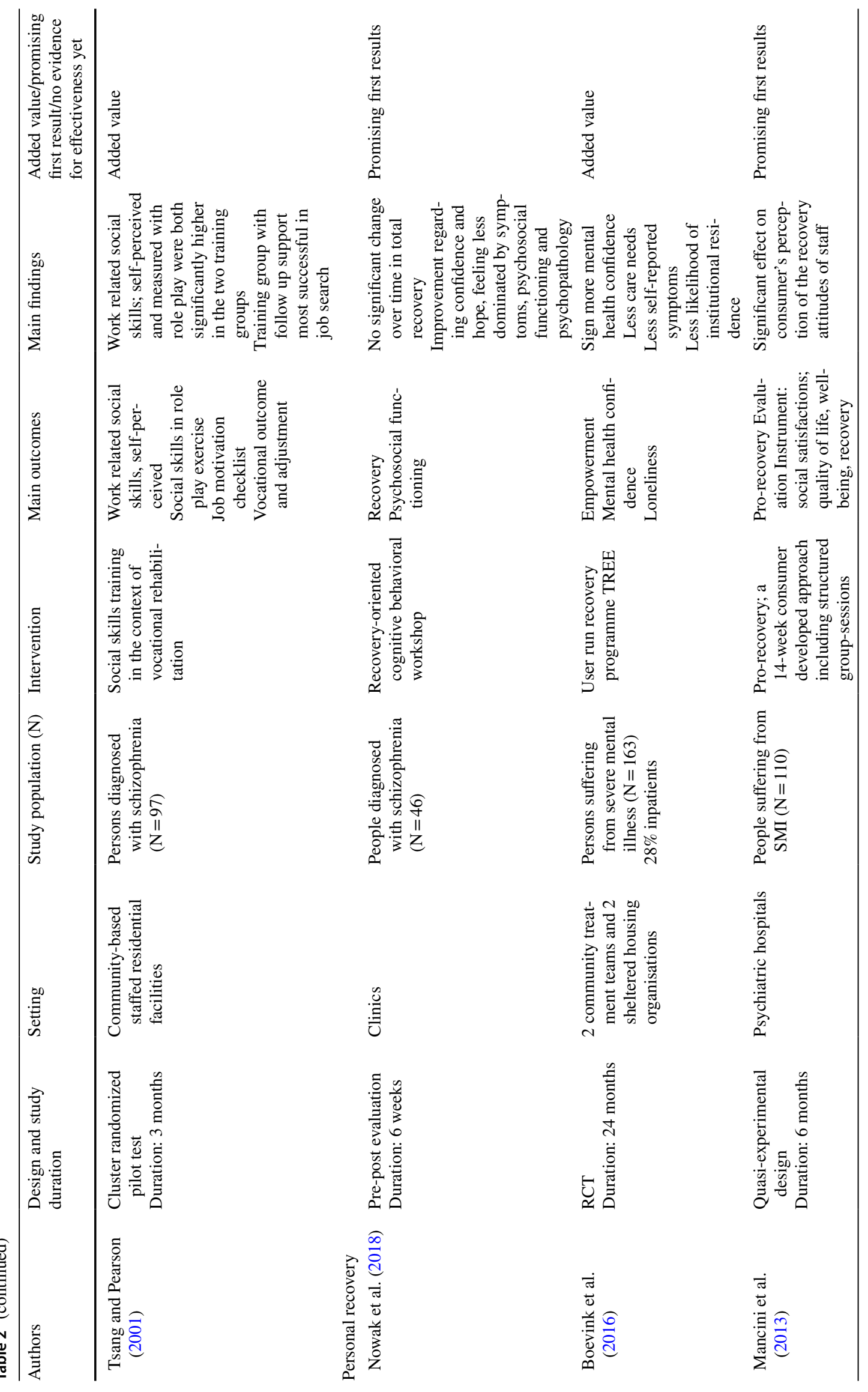




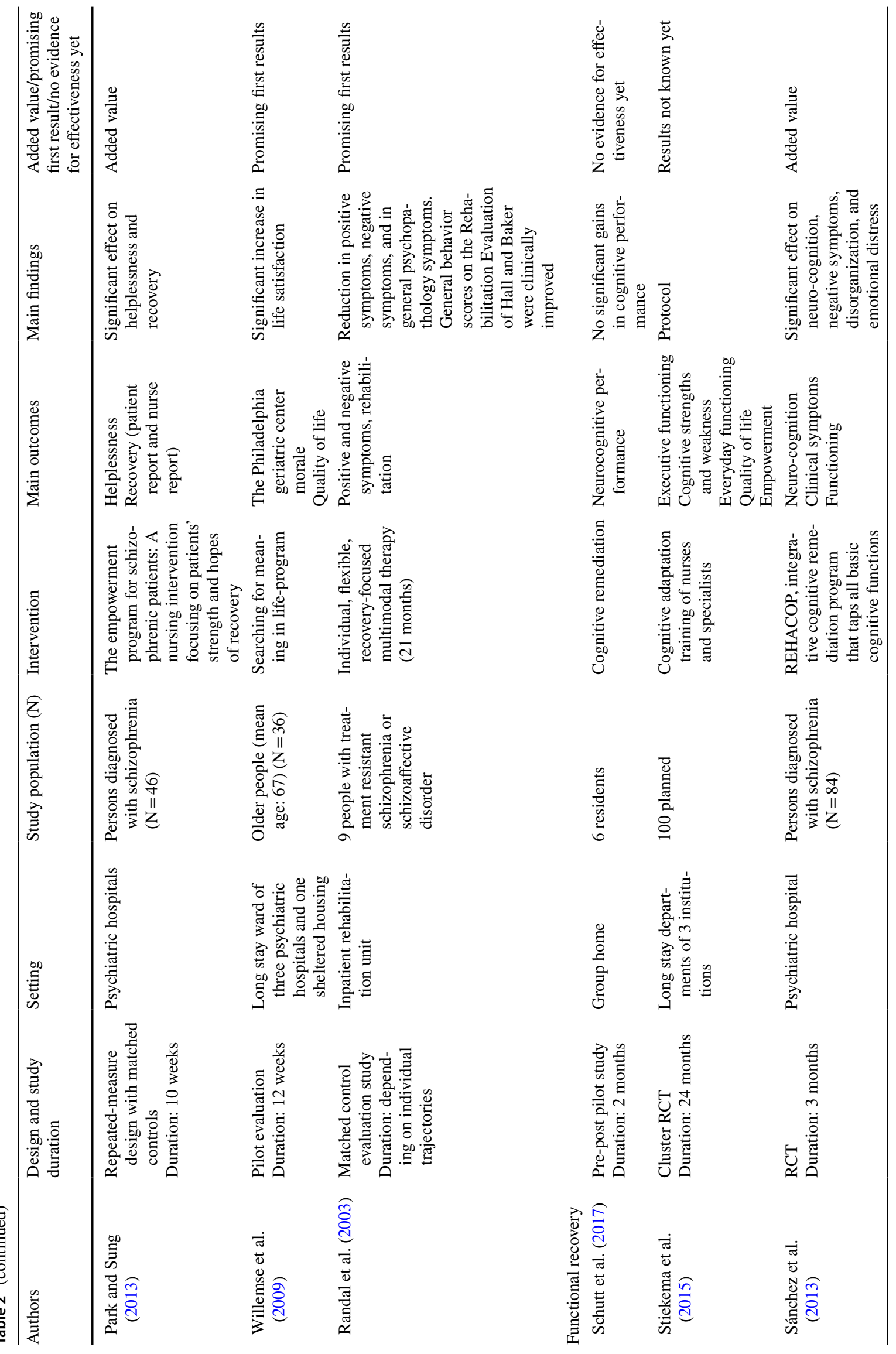




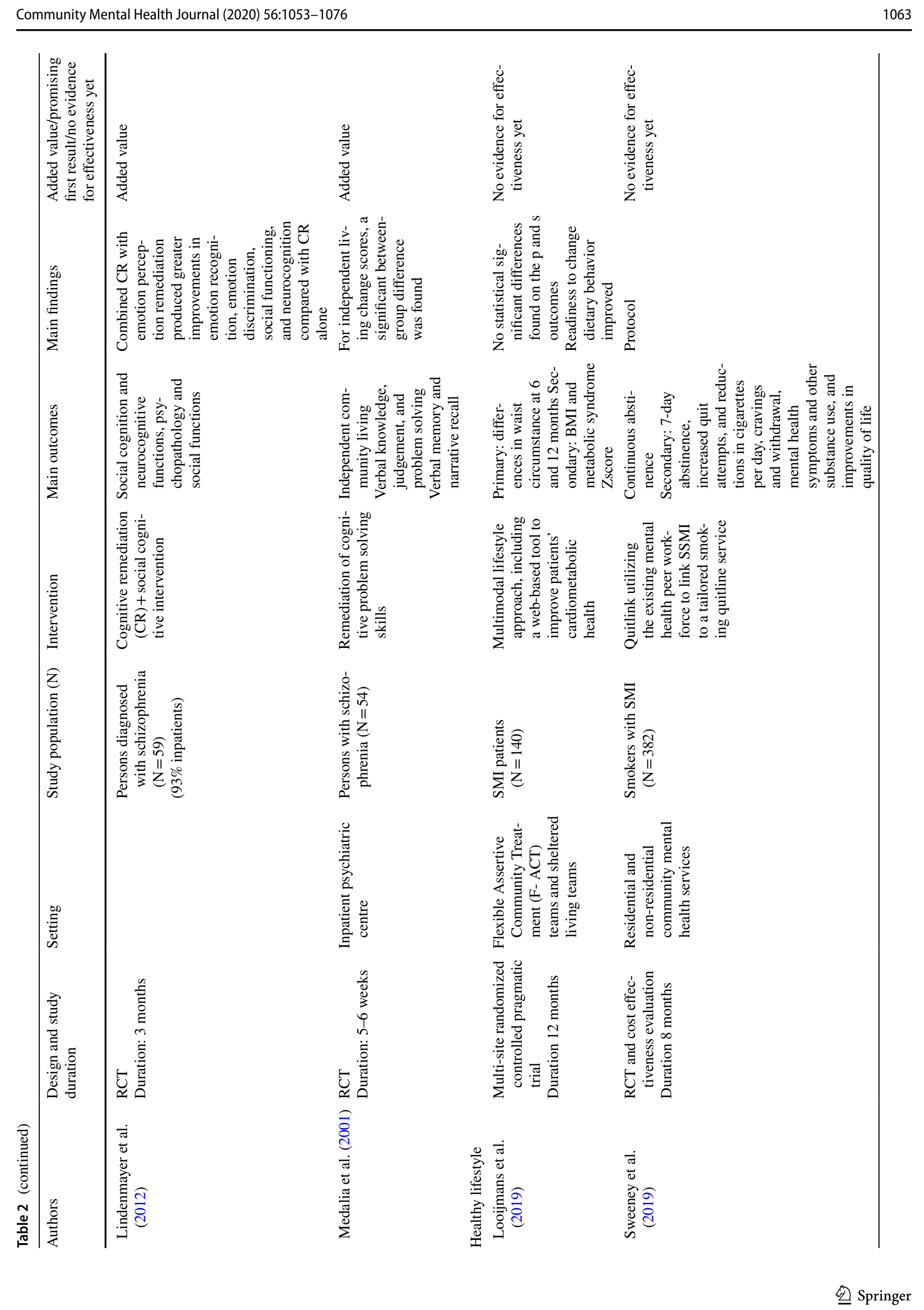




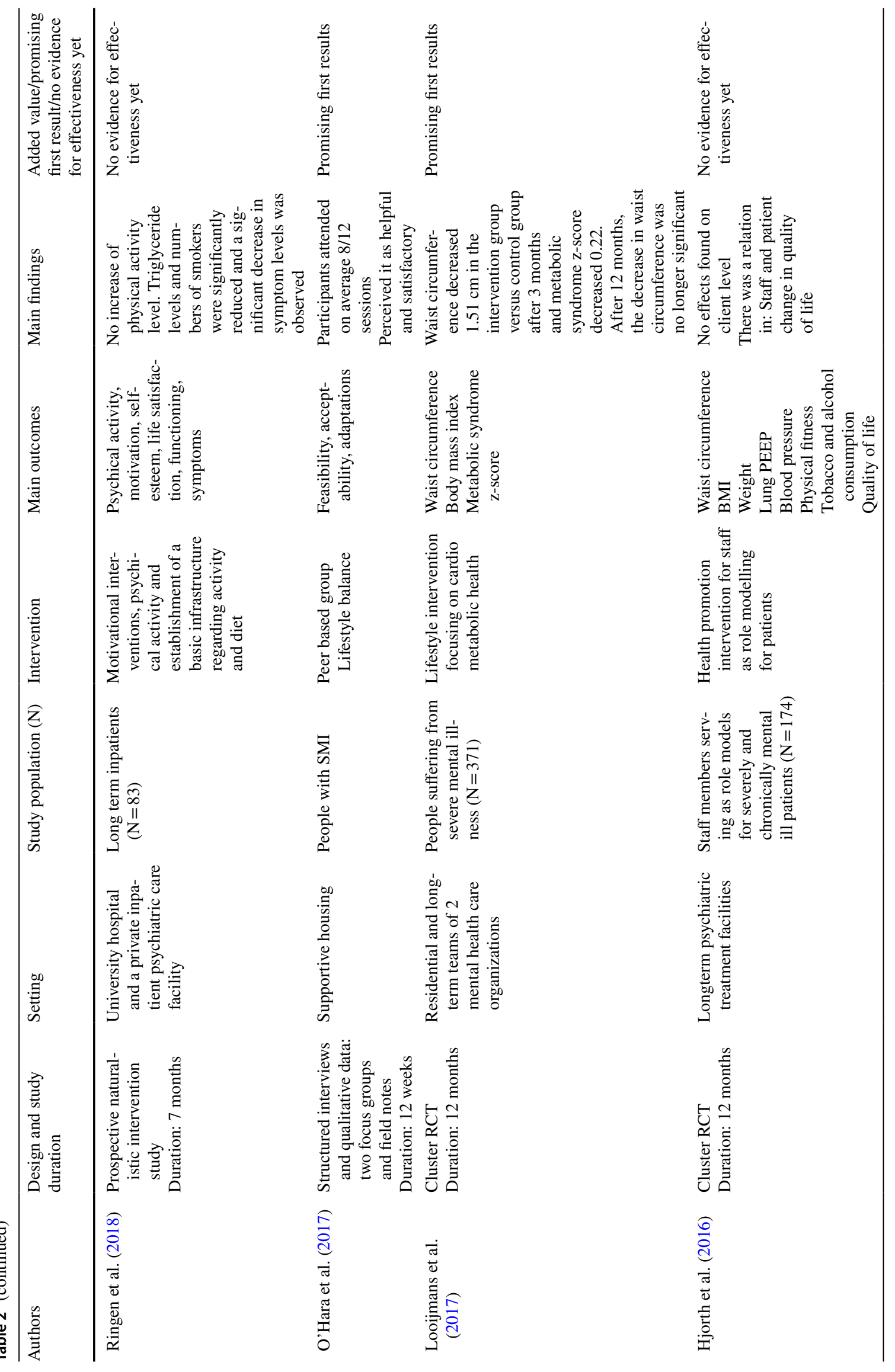




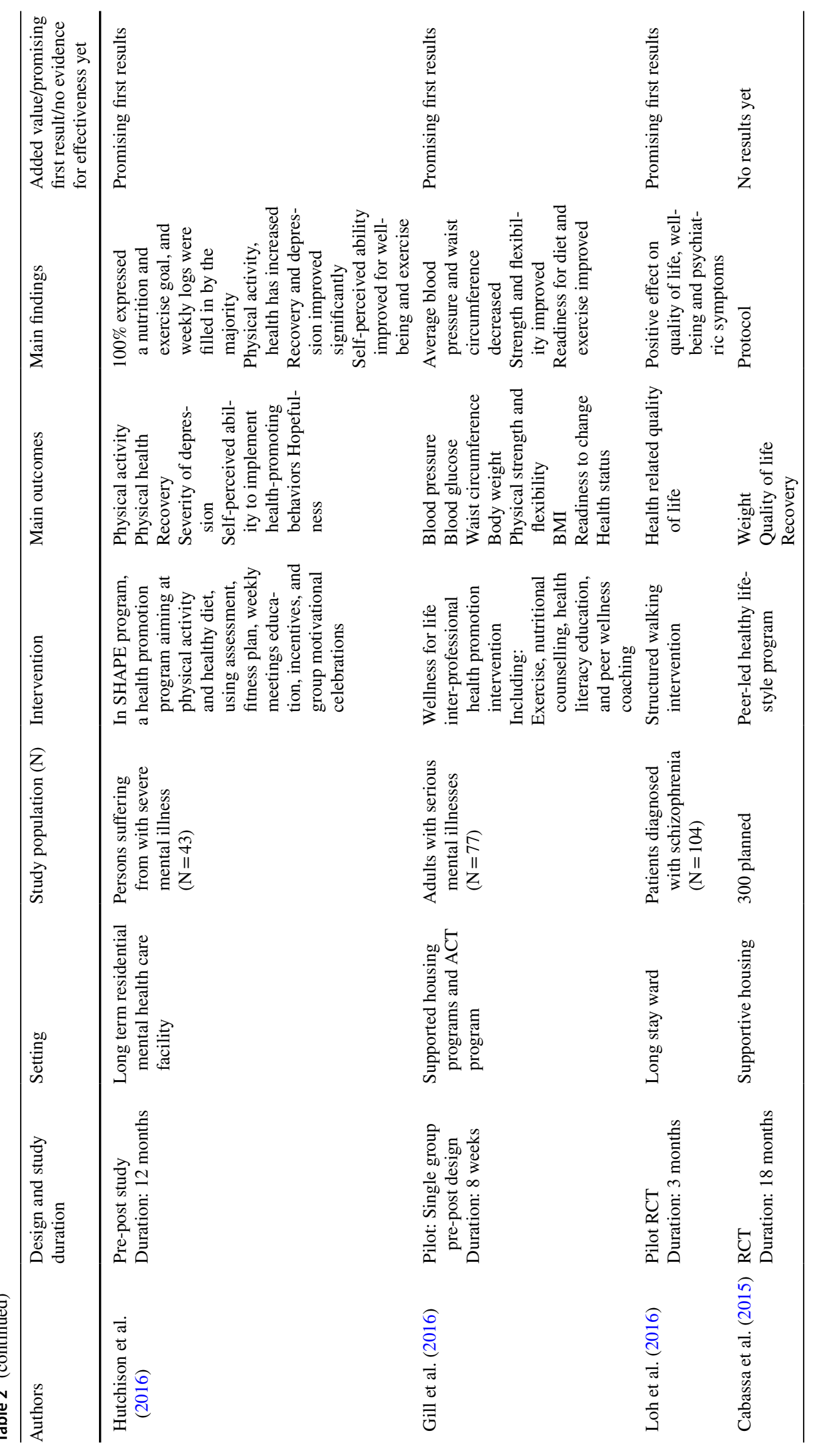




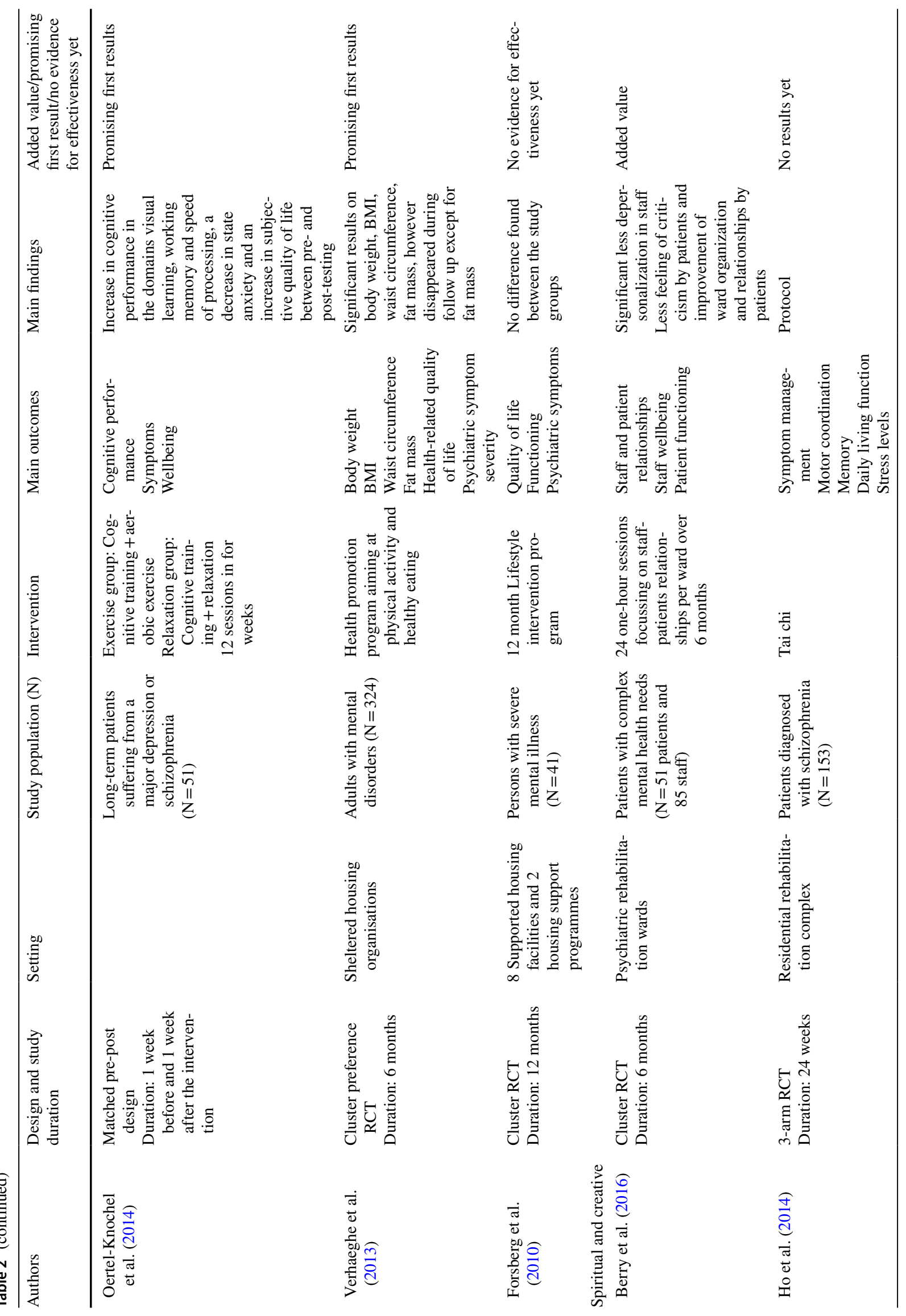




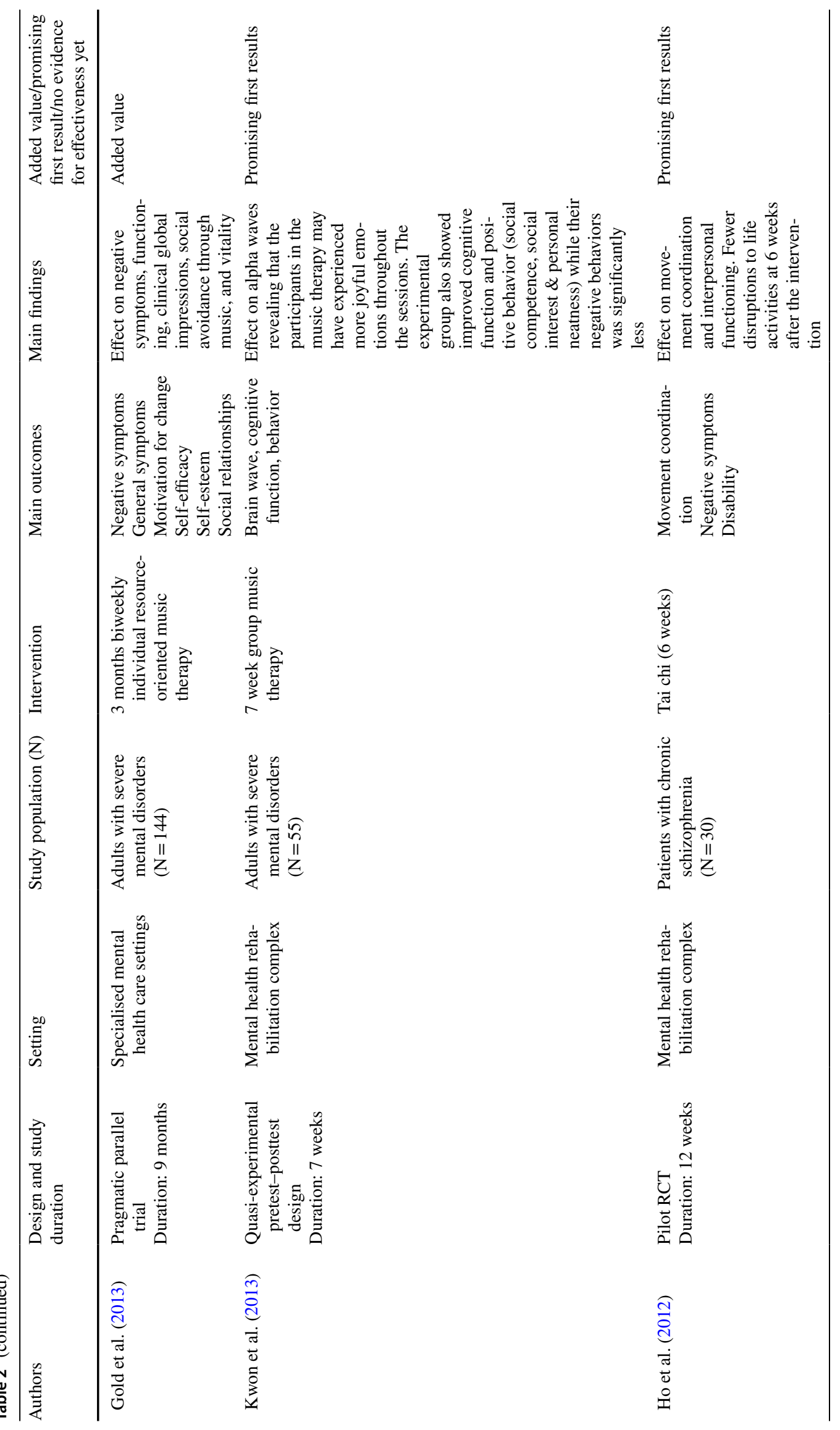




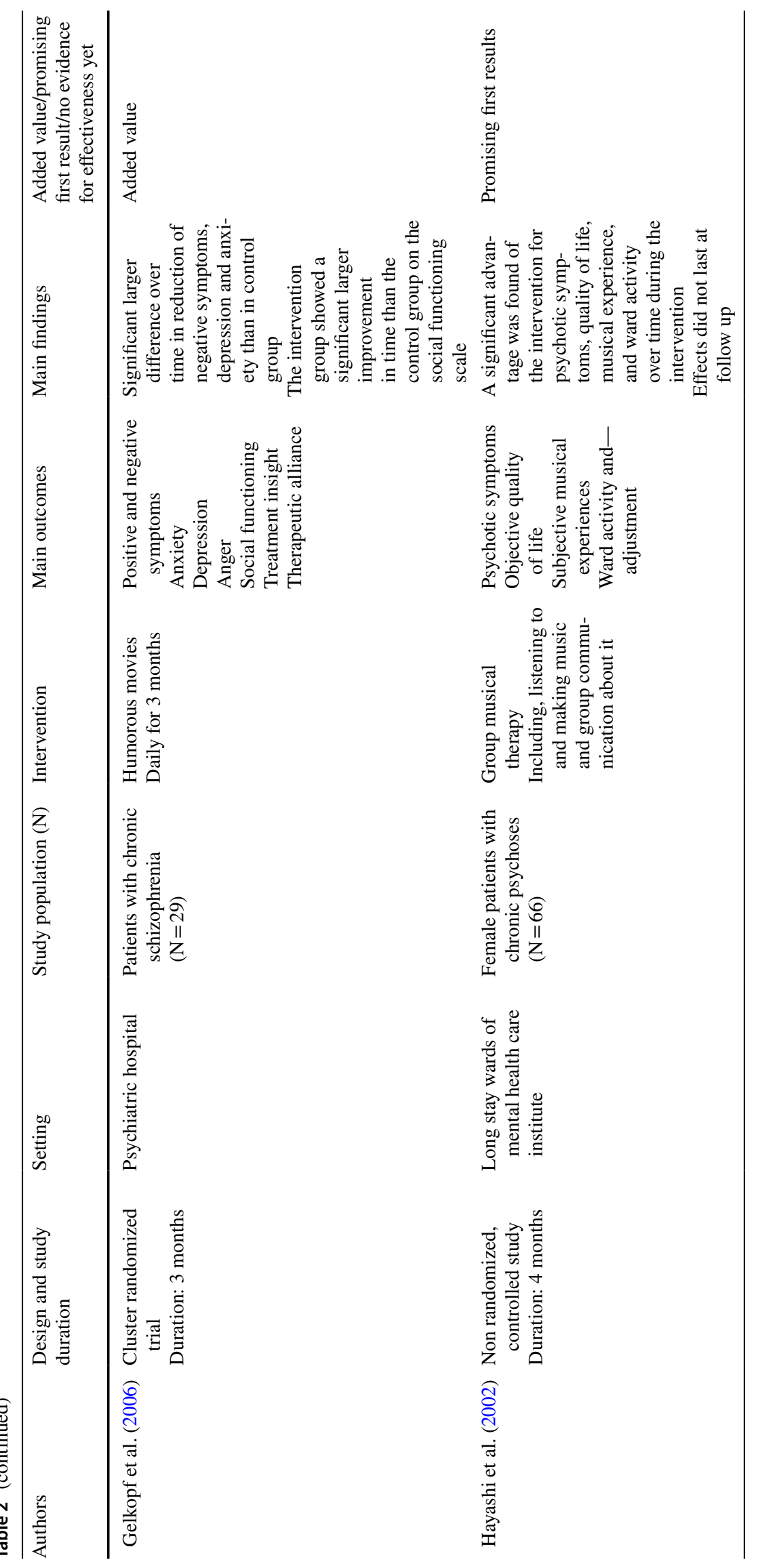


achievement, seven at achieving specific and/or disability management and two at vocational rehabilitation. One study concerned a staff-training program designed to increase patients' engagement.

Of the nine studies that evaluated interventions aimed at goal attainment, seven interventions were totally or partly based on the 'choose-get-keep' model (Anthony et al. 2014; Ellison et al. 2011; Fagan-Pryor et al. 2009; Magliano et al. 2016; Pioli et al. 2006; Sanches et al. 2015; Vandevooren et al. 2007). Three of the goal attainment studies were RCTs, and four were uncontrolled/pre-post design. Five of these studies showed (small) positive results (Ellison et al. 2011; Fagan-Pryor et al. 2009; Magliano et al. 2016; Pioli et al. 2006; Vandevooren et al. 2007), among others, concerning functioning and residential status. Bitter et al. (2017) evaluated, by means of a cluster randomized trial, CARe: A rehabilitation approach based on the strengths model and personal recovery in teams of supported accommodation, but did not find any differences in outcomes between the clients of trained and untrained teams.

Of the studies on interventions concerning skills and illness/disability management, two RCT studies evaluated the illness management and recovery (IMR) approach (Beentjes et al. 2018; Levitt et al. 2009). The Levitt study reported significant improvements in illness management, symptoms and psychosocial functioning, while the Beentjes' e-IMR study did not due to low implementation rates. Lindström et al. (2012) conducted a study on a home-based occupational therapy intervention aiming at daily occupations including remediation and compensatory strategies. The authors observed positive significant results on most outcomes (goal attainment, social interaction, satisfaction with daily occupations, activities of daily living (ADL) and psychiatric symptoms). Anzai et al. (2002) examined an RCT on an training program for illness management skills based on Liberman's community re-entry module, resulting in positive effects including knowledge and skills and community participation. In a small, pre-post study on a short educational training course on using the internet and touch screen, no effects were found on social isolation, self-esteem and internet use (Loi et al. 2016). Three studies (Park and Han 2018; Seo et al. 2007; Tsang and Pearson 2001) examined societal recovery explicitly focussed on social skills. Tsang and Pearson (2001) evaluated social skills training in the context of vocational rehabilitation. This cluster randomized pilot found positive results for work-related social skills, motivation to seek employment and success in job search. Seo et al. (2007) conducted a quasi-experimental study on social skills group training that included conservational and assertiveness skills based on the Liberman modules. The results showed a difference in improvement of social skills and selfesteem in favour of the intervention group. Park and Han (2018) studied with a quasi-experimental design a 5-week communication program based on communication theory of Walsh and existing of ten sessions. They found improved communication, and relational skills, but no improvement in problem solving, though used an alpha of 0.70 .

Two studies evaluated interventions aimed at vocational rehabilitation. Oka et al. (2004) evaluated a hybrid occupational therapy and supported employment intervention by means of a retrospective study. Positive results were achieved concerning social functioning and hospitalisation. Rogers et al. (2006) evaluated the choose-get-keep approach in a vocational context compared with enhanced state vocational rehabilitation and found no differences between the groups. A positive effect on vocational status was found for both interventions, indicating that a rehabilitation approach aiming at work can be effective for this group.

Finally, the remaining studies were concerned with client engagement in activities (Killaspy et al. 2015; Sheridan et al. 2018) and psychoeducation based on cognitive behavioral therapy (McMurran et al. 2011). Killaspy et al. (2015) evaluated a staff-training program designed to increase patients' engagement in activities. In this cluster-randomized trial, no differences were found between the study groups in engagement in activities. Sheridan et al. (2018) studied the effects of a supported socialisation volunteer partner group to stimulate social and leisure activities. In their qualitative thematic analyses of diary data they found indications for positive effects on involvement in normalising life, connectedness, physical health, social capacity and culture engagement. McMurran et al. (2011) published on a protocol to evaluate a 12-session group intervention aimed at problem solving.

\section{Personal Recovery}

The six studies in this category evaluated interventions aimed at personal recovery (including outcomes on empowerment, hope, confidence, and quality of life or comparable). All studies showed added value or promising first results. Of these studies, one was an RCT and five were semi-controlled or pre-post designs. Two studies were peer-run interventions. One of these peer-run interventions examined confidence and care needs (Boevink et al. 2016) and the other on consumers' perception of the recovery attitudes on the staff (Mancini et al. 2013).

One study focussed especially on elderly patients and showed a small but positive result concerning life satisfaction (Willemse et al. 2009). Park and Sung (2013) reported results of a study on a 6-week, recovery-oriented nursing intervention. This study also showed positive results on helplessness and recovery, but due to the non-controlled design, these results need further confirmation in replication studies. There were two studies on therapies to enhance personal recovery (Nowak et al. 2018; Randal et al. 2003). Randal 
et al. (2003) conducted a small, matched-control evaluation study on individual recovery-focused multimodal therapy. Following Evans' design hierarchy, the results can be interpreted as promising with outcomes showing significantly more improvement of positive and negative symptoms and a decrease of deviant behavior, e.g. verbal aggression and violence. Nowak et al. did a pre-post study on a recoveryoriented cognitive behavioral workshop of 6 weeks. They found no significant change in total recovery, but they did find significant improvements in sub scales including confidence, hope and psycho social functioning.

\section{Functional Recovery}

This category included five studies evaluating interventions focused on improvement of cognitive and executive functions. Four were RCTs, and one had a pre-post design. A study on an integrative program that focused on all basic cognitive functions showed positive results concerning vocational outcomes, family contact and social competence (Sánchez et al. 2013). Lindenmayer et al. (2012) conducted an RCT on an intervention that combined cognitive remediation with social cognition training. The combined intervention resulted in greater improvements in emotion recognition, emotion discrimination, social functioning and neuro-cognition compared with cognitive remediation alone. Another study resulting in interesting results was a cognitive remediation intervention focusing on problem solving skills (Medalia et al. 2001). This study found a significant difference for independent living. Schutt et al. (2017) executed a small pre-post study on a cognitive remediation intervention, but did not find relevant outcomes. Stiekema et al. (2015) published on their protocol to evaluate a cognitive adaptation training (CAT).

\section{Healthy Lifestyle}

We found thirteen studies focusing on lifestyle interventions; all were published after 2010. Seven were RCTs, five were semi-controlled or pre-post studies and one was qualitative. Seven of these studies showed promising first results, four did not show evidence and two were protocol papers. Loh et al. (2016) executed a (pilot) RCT on a structured walking intervention. In this study, the participants of the control group scored slightly better on quality of life, psychiatric symptoms, physical role limitations and physical functioning after 3 months. Hjorth et al. (2016) evaluated an intervention program for improving physical health in staff and its impact on patient's health. The intervention had a positive effect on the waist circumference and blood pressure for the staff, and there was a statistically significant association between the staff change in each facility and the patients' change in health parameters.
Looijmans et al. (2017) conducted a cluster RCT on lifestyle intervention that focused on cardio metabolic health. This intervention led to positive results after 3 months on waist circumstance and metabolic syndrome. The same research group studied the use of a web-based tool (Looijmans et al. 2019) in FACT teams and sheltered living teams. Findings indicate no significant improvements on the primary and secondary outcomes and an improvement on the readiness to change. Oertel-Knöchel et al. (2014) conducted a combined cognitive-aerobic/relaxation intervention showing that physical exercise is a valuable addition to cognitive training. Verhaeghe et al. (2013), Cabassa et al. (2015), Forsberg et al. (2010) and O'Hara et al. (2017) also all studied a lifestyle program. Verhaeghe et al. conducted a cluster RCT on a comprehensive lifestyle intervention (psycho-education, supervised exercise and individual support) in sheltered housing services. Although initially small positive results were achieved on weight, body mass index (BMI) and waist circumstances, these results almost all disappeared during follow-up. No differences were found regarding secondary outcomes (i.e., symptoms and quality of life). Cabassa et al. published a study protocol. Forsberg et al. did not find support for the added value. O'Hara et al. studied the results of a peer based group and did this qualitatively, using focus groups and field notes, and added this with structured interviews. The results indicate participants attended on average three quarter of the sessions and perceived them as helpful and satisfactory. Ringen et al. (2018), Hutchison et al. (2016) and Gill et al. (2016) all executed pre-post evaluations on a promotion / motivational program, of which the first did not show improvements and the latter two resulted in positive results on physical activity and physical health. Sweeney and Baker, finally, published two protocol papers on an intervention in which existing peer workers tailor clients to appropriate smoking quitline service (Sweeney et al. 2019).

\section{Spiritual and Creative Therapy}

This category contained seven studies. Two studies (one protocol) evaluated Tai chi (Ho et al. 2012, 2014) of which a pilot RCT showed promising results concerning movement and interpersonal functioning. Three studies (Gold et al. 2013; Hayashi et al. 2002; Kwon et al. 2013) evaluated a form of music therapy. In all studies, positive results were achieved concerning amount others: Negative symptoms (Gold et al. 2013), cognitive function (Hayashi et al. 2002), positive behavior (Kwon et al. 2013), and quality of life (Hayashi et al. 2002). These positive results, however, did not last through the last follow-up.

One study in this category evaluated the effect of watching humorous movies. Watching these movies regularly for 3 months appeared to have a small positive effect on negative symptoms, depression and anxiety, and social competence 
(Gelkopf et al. 2006). The seventh study was a cluster trial on a ward intervention to improve patient-staff relationships and wellbeing leading to significant differences in depersonalization in staff and criticism experienced by clients (Berry et al. 2016).

\section{Discussion}

With this study, we aimed to achieve insight into which psychosocial interventions are available to support recovery in other dimensions than the clinical one and evaluated in people with SMI who live in supported accommodations. Additionally, we explored what scientific knowledge is available about the outcomes of these interventions. We found 53 studies with different types of interventions aiming at several non-clinical dimensions of recovery. Almost a quarter $(22.6 \%)$ of these interventions showed added value and almost half of them (47.2\%) first promising results. This is a hopeful result that shows that improvement on recovery is possible, even for people with SMI living in supported accommodations which are, as shown in the introduction, often dealing with long-term and complex needs. The articles included in this study provide knowledge concerning the current use of psychosocial interventions in supported accommodations and give us new insights in the opportunities for implementation, further development and evaluation of interventions.

These findings indicate that there have been some practice and research attention for the other dimensions of recovery for the group of people who need supported accommodation in the last 20 years. Interventions aimed at societal recovery have the longest tradition in general mental healthcare, which is reflected in the larger number of papers found for the group living in supported accommodations and their publication date as well. Of these, most interventions were based on the Boston choose-get-keep rehabilitation approach which showed inconsistent results, some no added value, some promising results. Further study needs to bring answers to when, for whom and why these interventions do or do not work. Realist evaluations are the most suiting design for this (Wong et al. 2016). Interventions showing the most consistent added value included IMR and social and self-management skills trainings and are therefore relevant to follow and replicate.

Additionally, we found small amounts of papers concerning the two other known recovery dimensions: personal and functional recovery. Developments which are relevant to follow and replicate if we truly want the whole group with SMI to profit from the paradigm shift in mental health care towards a broader definition of recovery in which more recognition exists for the personal experience of people with mental illnesses (Leamy et al. 2011). On personal recovery, markedly, all six interventions found had added value or promising results. Noteworthy are the two interventions with added value: the TREE peer-to-peer intervention and the empowerment program provided by nurses. Of the five functional recovery interventions, three showed added value, which all included cognitive remediation interventions. Cognitive adaptation training have not been studied frequently, but is one to follow: it is in concept easy to implement and if effective, a large contribution to independent functioning can be expected. Interventions on the functional recovery dimension are especially relevant when considering that cognitive dysfunction and related negative symptoms can be strong obstructing factors in the life of people with severe mental health problems (Quee et al. 2014; Stiekema et al. 2016).

Additional to the known recovery dimensions, we found a relatively large number of studies on healthy lifestyle (13) and on the spiritual and creative domain (7). Healthy lifestyle is a relevant life area as a substantial number of people suffering from a severe mental illness are affected by comorbid medical conditions which influence their life expectancy, quality of life and recovery on other dimensions (Scott and Happell 2011). No interventions showed added value, but half of them were promising. Noteworthy is that most of the health promotion interventions, all including exercise and some a healthy diet as well, showed promising results. Interesting was the structured walking intervention showing promising results, which seems an easy to implement intervention with large impact. Five interventions: the peer led, smoking, web-based, and the two health promotion/ motivational interventions by staff did not show added value. The results indicate that concrete lifestyle programs might add more to the results.

In the spiritual and creative intervention category three music therapies were studied of which, noteworthy, one showed added value and two promising results. Tai chi was twice studied as intervention: one showing no results and one promising results. Markedly, humorous movie watching as intervention showed added value. This finding relates to current insights: cultural interventions have high potential for health gains as was recently underlined in a scoping review of the WHO (Fancourt and Finn 2019).

This broader scope and promising results are hopeful developments especially as people with severe mental illness experience several unmet needs (Bitter et al. 2016; de Heer-Wunderink 2012; Wiersma 2006). However, compared with the ambulatory treated people with mental illness, the number of studies we found on recovery can still be considered relatively low (van Weeghel et al. 2019b). This is not surprising because since the start of deinstitutionalisation in the second half of the twentieth century, the focus of practice, research and policy increasingly shifted towards the development of ambulant and community-oriented services (Burns and Firn 2017). Although this was an important 
development in mental health care, which led to the increasing opportunity for people with SMI to participate in society, the risk exists that a knowledge gap emerges concerning the group in need supported accommodation (McPherson et al. 2018). It is therefore important that more studies focus on this group to gain more insight in what these people need in their recovery and to develop interventions that match their needs.

\section{Strengths and Weaknesses of the Study}

This study has several strengths and limitations. A strength was the broad scope. Our aim was to provide an impression of psychosocial interventions that exist for people with SMI who need supported accommodation and to provide first insights into what is known about the effectiveness of these interventions. Therefore, we used a broad search strategy and included a variety of interventions aiming at a broad range of outcomes and executed in different settings and (international) contexts, and included all types of study designs. When developments in the recovery field are a bit further along, a quantitative synthesis would add to our knowledge. At this point the number of studies in the supported accommodation field is too small yet to perform a quantitative review (Chilvers et al. 2006; McPherson et al. 2018). A point of attention is that we used information provided in the included articles only, which sometimes was somewhat poor, for example not all papers published effect sizes. So, it might be that the quality of some papers is displayed more positively if it was based on the $p$ values only. Another note is that when performing a review, a selection of specific search terms is chosen. There is always a risk that not all relevant papers end up in the results due to word use in titles, abstracts and key words. When reading this and other reviews, this should be kept in mind. Nevertheless, this study provides a broad overview of interventions on several dimensions of recovery besides the clinical one that can give supported accommodation an impression of interventions that may be relevant and sufficient to implement, and bring the recovery forward, even in people that cope with severe, and therefore often complex and long-term, mental illnesses.

\section{Suggestions for Development of Practice and Research}

Research specifically focussing on the recovery dimensions besides clinical recovery of people with severe mental illness who live in supported accommodations remains limited but seems to be in development. We also can conclude that a broader vision towards recovery in these settings has gained attention and that, regarding all other dimensions of recovery, hopeful results have been achieved so far.

Four challenges can be appointed concerning the practice and research of interventions for people with severe mental illness who live in supported accommodations. The first challenge is the further development and professionalization of recovery-oriented care and support offered for this specific group of people. Effective and promising interventions should be developed and made available for all people with severe mental illness, despite their place in the care landscape (Couwenbergh and van Weeghel 2014).

The second challenge is to accompany developments in practice with research to gain more insight into what works, for whom and what does not, so that the provided care can be more personalized. Specific knowledge is needed concerning the group of people who are in need of supported accommodation. For example, we were surprised that for some well-known recovery interventions, for example, the wellness recovery action plan (WRAP) (Fukui et al. 2011) or narrative enhancement and cognitive therapy (NECT) (Fukui et al. 2011), no studies were found explicitly focussing on people living in supported accommodation. Here may lay a chance for further development, as it is worthwhile to study interventions that have proved themselves in ambulant contexts to see if they also can help clients with more complex and supported living needs.

The third challenge is the integration of different approaches towards recovery. In several countries, different forms of support are fragmentized (Boevink et al. 2016). For example, in the Netherlands a separation exists between clinical mental health care services and supported accommodation services. The insight is growing that integration of different aspects of recovery may lead to better outcomes (Corrigan et al. 2012). This might lead to improvement of recovery orientation of the care for people living in supported accommodation. Altogether, it is recommended that supported accommodation services reconsider their scope and position in the care landscape and consider broadening and strengthening their recovery-oriented services, as well as stronger collaborations between stakeholders including mental health treatment providers, supported housing organisations and local organizations for community support.

The fourth challenge is the professionals' interest, knowledge and implementation skills to adapt and use state-of-the-art interventions. Working evidence based asks for an innovative mind set as well as time and support in keeping up-to-date and using new interventions that were proven effective in research. 
Funding The funding was provided by Five supported housing organisations and Storm Rehabilitation.

Open Access This article is licensed under a Creative Commons Attribution 4.0 International License, which permits use, sharing, adaptation, distribution and reproduction in any medium or format, as long as you give appropriate credit to the original author(s) and the source, provide a link to the Creative Commons licence, and indicate if changes were made. The images or other third party material in this article are included in the article's Creative Commons licence, unless indicated otherwise in a credit line to the material. If material is not included in the article's Creative Commons licence and your intended use is not permitted by statutory regulation or exceeds the permitted use, you will need to obtain permission directly from the copyright holder. To view a copy of this licence, visit http://creativecommons.org/licenses/by/4.0/.

\section{References}

Anthony, W. A. (1990s). Recovery from mental illness: The guiding vision of the mental health service system in the 1990s. Psychosocial Rehabilitation Journal, 16(4), 11.

Anthony, W. A., Cohen, M. R., Farkas, M., \& Gagne, C. (2002). Psychiatric rehabilitation (2nd ed.). Boston: Boston University, Center for Psychiatric Rehabilitation.

Anthony, W. A., Ellison, M. L., Rogers, E. S., Mizock, L., \& Lyass, A. (2014). Implementing and evaluating goal setting in a statewide psychiatric rehabilitation program. Rehabilitation Counseling Bulletin, 57(4), 228-237.

Anzai, N., Yoneda, S., Kumagai, N., Nakamura, Y., Ikebuchi, E., \& Liberman, R. P. (2002). Rehab rounds: Training persons with schizophrenia in illness self-management: A randomized controlled trial in Japan. Psychiatric Services, 53(5), 545-547.

Arksey, H., \& O'Malley, L. (2005). Scoping studies: Towards a methodological framework. International Journal of Social Research Methodology, 8(1), 19-32.

Beentjes, T. A. A., Goossens, P. J. J., Vermeulen, H., Teerenstra, S., Nijhuis-van der Sanden, M. W. G., \& van Gaal, B. G. I. (2018). E-IMR: E-health added to face-to-face delivery of Illness Management \& Recovery programme for people with severe mental illness, an exploratory clustered randomized controlled trial. BMC Health Services Research, 18(1), 962.

Berry, K., Haddock, G., Kellett, S., Roberts, C., Drake, R., \& Barrowclough, C. (2016). Feasibility of a ward-based psychological intervention to improve staff and patient relationships in psychiatric rehabilitation settings. British Journal of Clinical Psychiatry, 55(3), 236-252.

Bitter, N. A., Roeg, D. P. K., van Nieuwenhuizen, Ch., \& van Weeghel, J. (2016). Identifying profiles of service users in housing services and exploring their quality of life and care needs. BMC Psychiatry, 16(1), 419.

Bitter, N. A., Roeg, D. P. K., van Assen, M. A. L. M., van Nieuwenhuizen, Ch., \& van Weeghel, J. (2017). How effective is the comprehensive approach to rehabilitation (CARe) methodology? A cluster randomized controlled trial. BMC Psychiatry, 17(1), 396.

Boevink, W., Kroon, H., van Vugt, M., Delespaul, P., \& van Os, J. (2016). A user-developed, user run recovery programme for people with severe mental illness: A randomised control trial. Psychosis, 8(4), 287-300.

Burns, T., \& Firn, M. (2017). Outreach in community mental health care: A manual for practitioners. Oxford: Oxford University Press.
Burns, T., Catty, J., Becker, T., Drake, R. E., Fioritti, A., Knapp, M., et al. (2007). The effectiveness of supported employment for people with severe mental illness: A randomised controlled trial. The Lancet, 370(9593), 1146-1152.

Cabassa, L. J., Stefancic, A., O’Hara, K., El-Bassel, N., Lewis-Fernández, R., Luchsinger, J. A., et al. (2015). Peer-led healthy lifestyle program in supportive housing: Study protocol for a randomized controlled trial. Trials, 16, 388 .

Chilvers, R., Macdonald, G. M., \& Hayes, A. A. (2006). Supported housing for people with severe mental disorders. Cochrane Database of Systematic Reviews, 4, CD000453.

Corrigan, P. W., Mueser, K. T., Bond, G. R., Drake, R. E., \& Solomon, P. (2012). Principles and practice of psychiatric rehabilitation: An empirical approach. New York: Guilford Press.

Couwenbergh, C., \& van Weeghel, J. (2014). Crossing the bridge: National action plan to improve care of severe mental illness. Phrenos, Center of expertise in Utrecht.

Davidson, L., Borg, M., Marin, I., Topor, A., Mezzina, R., \& Sells, D. (2005). Processes of recovery in serious mental illness: Findings from a multinational study. American Journal of Psychiatric Rehabilitation, 8(3), 177-201.

de Heer-Wunderink, C. (2012). Successful community living: A 'Utopia'? A survey of people with severe mental illness in Dutch Regional Institutes for Residential Care. Groningen.

de Heer-Wunderink, C., Visser, E., Caro-Nienhuis, A., Sytema, S., \& Wiersma, D. (2012a). Supported housing and supported independent living in the Netherlands, with a comparison with England. Community Mental Health Journal, 48(3), 321-327.

de Heer-Wunderink, C., Visser, E., Sytema, S., \& Wiersma, D. (2012b). Social inclusion of people with severe mental illness living in community housing programs. Psychiatric Services, 63(11), 1102-1107.

Ellison, M. L., Rogers, E. S., Lyass, A., Massaro, J., Wewiorski, N. J., Hsu, S. T., et al. (2011). Statewide initiative of intensive psychiatric rehabilitation: Outcomes and relationship to other mental health service use. Psychiatric Rehabilitation Journal, 35(1), 9.

Evans, D. (2003). Hierarchy of evidence: A framework for ranking evidence evaluating healthcare interventions. Journal of Clinical Nursing, 12(1), 77-84.

Fagan-Pryor, E. C., Haber, L. C., Harlan, D., \& Rumple, S. (2009). The impact of a recovery-based program on veterans with long inpatient psychiatric stays. Issues in Mental Health Nursing, 30(6), 372-376.

Fancourt, D., \& Finn, S. (2019). What is the evidence on the role of the arts in improving health and well-being? A scoping review. Retrieved from https://apps.who.int/iris/bitstream/handle/10665 /329834/9789289054553-eng.pdf.

Farkas, M., \& Anthony, W. A. (2010). Psychiatric rehabilitation interventions: A review. International Review of Psychiatry, 22(2), 114-129.

Forsberg, K. A., Björkman, T., Sandman, P. O., \& Sandlund, M. (2010). Influence of a lifestyle intervention among persons with a psychiatric disability: A cluster randomised controlled trail on symptoms, quality of life and sense of coherence. Journal of Clinical Nursing, 19(11-12), 1519-1528.

Fox, J., \& Horan, L. (2016). Individual perspectives on the Wellness Recovery Action Plan (WRAP) as an intervention in mental health care. International Journal of Psychosocial Rehabilitation, 20(2), 110-125.

Fukui, S., Starnino, V. R., Susana, M., Davidson, L. J., Cook, K., Rapp, C. A., et al. (2011). Effect of Wellness Recovery Action Plan (WRAP) participation on psychiatric symptoms, sense of hope, and recovery. Psychiatric Rehabilitation Journal, 34(3), 2014.

Gelkopf, M., Gonen, B., Kurs, R., Melamed, Y., \& Bleich, A. (2006). The effect of humorous movies on inpatients with chronic 
schizophrenia. The Journal of Nervous and Mental Disease, 194(11), 880-883.

Gill, K. J., Zechner, M., Zambo Anderson, E., Swarbrick, M., \& Murphy, A. (2016). Wellness for life: A pilot of an interprofessional intervention to address metabolic syndrome in adults with serious mental illnesses. Psychiatric Rehabilitation Journal, 39(2), 147.

Gold, C., Mössler, K., Grocke, D., Heldal, T. O., Tjemsland, L., Aarre, T., et al. (2013). Individual music therapy for mental health care clients with low therapy motivation: Multicentre randomised controlled trial. Psychotherapy and Psychosomatics, 82(5), 319-331.

Hansen, J. P., Østergaard, B., Nordentoft, M., \& Hounsgaard, L. (2012). Cognitive adaptation training combined with assertive community treatment: A randomised longitudinal trial. Schizophrenia Research, 135(1), 105-111.

Hayashi, N., Tanabe, Y., Nakagawa, S., Noguchi, M., Iwata, C., Koubuchi, Y., et al. (2002). Effects of group musical therapy on inpatients with chronic psychoses: A controlled study. Psychiatry and Clinical Neurosciences, 56(2), 187-193.

Hjorth, P., Davidsen, A., Kilian, R., Jensen, S. O. W., \& MunkJørgensen, P. (2016). Intervention to promote physical health in staff within mental health facilities and the impact on patients' physical health. Nordic Journal of Pyschiatry, 70(1), 62-71.

Ho, R. T. H., Au Yeung, F. S., Lo, P. H., Law, K. Y., Wong, K. O., Cheung, I. K., et al. (2012). Tai-chi for residential patients with schizophrenia on movement coordination, negative symptoms, and functioning: A pilot randomized controlled trial. EvidenceBased Complementary and Alternative Medicine. https://doi. org/10.1155/2012/923925.

Ho, R. T. H., Wan, A. H. Y., Au-Yeung, F. S. W., Lo, P. H. Y., Siu, P. J. C. Y., Wong, C. P. K., et al. (2014). The psychophysiological effects of tai-chi and exercise in residential schizophrenic patients: A 3-arm randomized controlled trial. BMC Complementary and Alternative Medicine, 14(1), 364.

Hutchison, S. L., Terhorst, L., Murtaugh, S., Gross, S., Kogan, J. N., \& Shaffer, S. L. (2016). Effectiveness of a staff promoted wellness program to improve health in residents of a mental health long-term care facility. Issues in Mental Health Nursing, 37(4), 257-264.

Ibrahim, N., Michail, M., \& Callaghan, P. (2014). The strengths based approach as a service delivery model for severe mental illness: A meta-analysis of clinical trials. BMC Psychiatry, 14(1), 243

Keet, R., de Vetten-Mc Mahon, M., Shields-Zeeman, L., Ruud, T., van Weeghel, J., Mulder, C. L., et al. (2019). Recovery for all in the community; position paper on principles and key elements of community-based mental health care. BMC Psychiatry. https:// doi.org/10.1186/s12888-019-2162-z.

Killaspy, H. (2016). Supported accommodation for people with mental health problems. World Psychiatry, 15(1), 74-75.

Killaspy, H., Marston, L., Green, N., Harrison, I., Lean, M., Cook, S., et al. (2015). Clinical effectiveness of a staff training intervention in mental health inpatient rehabilitation units designed to increase patients' engagement in activities (the Rehabilitation Effectiveness for Activities for Life [REAL] study): Single-blind, cluster-randomised controlled trial. The Lancet Psychiatry, 2(1), 38-48.

Killaspy, H., Marston, L., Green, N., Harrison, I., Lean, M., Holloway, F., et al. (2016). Clinical outcomes and costs for people with complex psychosis; a naturalistic prospective cohort study of mental health rehabilitation service users in England. BMC Psychiatry, 16(1), 95.

Kwon, M., Gang, M., \& Oh, K. (2013). Effect of the group music therapy on brain wave, behavior, and cognitive function among patients with chronic schizophrenia. Asian Nursing Research, 7(4), 168-174.

Leamy, M., Bird, V., Le Boutillier, C., Williams, J., \& Slade, M. (2011). Conceptual framework for personal recovery in mental health: Systematic review and narrative synthesis. The British Journal of Psychiatry, 199(6), 445-452.

Leff, H. S., Chow, C. M., Pepin, R., Conley, J., Ph, B., Allen, I. E., \& Seaman, C. A. (2015). Does one size fit all? What we can and can't learn from a meta-analysis of housing models for persons with mental illness. Psychiatric Services, 60(4), 473-482.

Levitt, A. J., Mueser, K. T., DeGenova, J., Lorenzo, J., Bradford-Watt, D., Barbosa, A., et al. (2009). Randomized controlled trial of illness management and recovery in multiple-unit supportive housing. Psychiatric Services, 60(12), 1629-1636.

Liberman, R. P., Kopelowicz, A., Ventura, J., \& Gutkind, D. (2002). Operational criteria and factors related to recovery from schizophrenia. International Review of Psychiatry, 14(4), 256-272.

Lindenmayer, J.-P., McGurk, S. R., Khan, A., Kaushik, S., Thanju, A., Hoffman, L., et al. (2012). Improving social cognition in schizophrenia: A pilot intervention combining computerized social cognition training with cognitive remediation. Schizophrenia Bulletin, 39(3), 507-517.

Lindström, M., Hariz, G. M., \& Bernspång, B. (2012). Dealing with real-life challenges: Outcome of a home-based occupational therapy intervention for people with severe psychiatric disability. OTJR: Occupation, Participation and Health, 32(2), 5-14.

Lloyd-Evans, B., Mayo-Wilson, E., Harrison, B., Istead, H., Brown, E., Pilling, S., et al. (2014). A systematic review and meta-analysis of randomised controlled trials of peer support for people with severe mental illness. BMC Psychiatry, 14(1), 39.

Loh, S. Y., Abdullah, A., Bakar, A. K. A., Thambu, M., \& Jaafar, N. R. N. (2016). Structured walking and chronic institutionalized schizophrenia inmates: A pilot rct study on quality of life. Global Journal of Health Science, 8(1), 238.

Loi, S. M., Hodson, S., Huppert, D., Swan, J., Mazur, A., \& Lautenschlager, N. T. (2016). Can a short internet training program improve social isolation and self-esteem in older adults with psychiatric conditions? International Psychogeriatrics, 28(10), 1737-1740.

Looijmans, A., Stiekema, A. P. M., Bruggeman, R., Van der Meer, L., Stolk, R. P., Schoevers, R. A., et al. (2017). Changing the obesogenic environment to improve cardiometabolic helath in residential patients with a severe mental illness: Cluster randomised controlled trial. The British Journal of Psychiatry, 211(5), 296-303.

Looijmans, A., Jörg, F., Bruggeman, R., Schoevers, R. A., \& Corpeleijn, E. (2019). Multimodal lifestyle intervention using a webbased tool to improve cardiometabolic health in patients with serious mental illness: Results of a cluster randomized controlled trial (LION). BMC Psychiatry, 19(1), 339.

Magliano, L., Puviani, M., Rega, S., Marchesini, N., Rossetti, M., \& Starace, F. (2016). Feasibility and effectiveness of a combined individual and psychoeducational group intervention in psychiatric residential facilities: A controlled, non-randomized study. Psychiatry Research, 235, 19-28.

Mancini, M. A., Linhorst, D. M., Menditto, A. A., \& Coleman, J. (2013). Statewide implementation of recovery support groups for people with serious mental illness: A multidimensional evaluation. The Journal of Behavioral Health Services \& Research, 40(4), 391-403.

Marder, S. R., Wirshing, W. C., Mintz, J., McKenzie, J., Johnston, K., Eckman, T. A., et al. (1996). Two-year outcome of social skills training and group psychotherapy for outpatients with schizophrenia. American Journal of Psychiatry, 153(12), 1585-1592.

McMurran, M., Crawford, M. J., Reilly, J. G., McCrone, P., Moran, P., Williams, H., et al. (2011). Psycho-education with problem solving (PEPS) therapy for adults with personality disorder: A 
pragmatic multi-site community-based randomised clinical trial. Trials, 12, 198.

McPherson, P., Krotofil, J., \& Killaspy, H. (2018). Mental health supported accommodation services: A systematic review of mental health and psychosocial outcomes. BMC Psychiatry, 18(1), 128.

Medalia, A., Revheim, N., \& Casey, M. (2001). The remediation of problem-solving skills in schizophrenia. Schizophrenia Bulletin, 27(2), 259.

Michon, H., van Busschbach, J., van Vugt, M., Stant, A., Kroon, H., Wiersma, D., \& van Weeghel, J. (2011). Effectiveness of the Individual Placement and Support (IPS) model of vocational rehabilitation for people with severe mental illnesses in the Netherlands. Psychiatrische Praxis, 38(S 01), OP26_EC.

Mueser, K. T., Meyer, P. S., Penn, D. L., Clancy, R., Clancy, D. M., \& Salyers, M. P. (2006). The illness management and recovery program: Rationale, development, and preliminary findings. Schizophrenia Bulletin, 32(suppl 1), S32-S43.

Mueser, K. T., Pratt, S. I., Bartels, S. J., Swain, K., Forester, B., Cather, C., \& Feldman, J. (2010). Randomized trial of social rehabilitation and integrated health care for older people with severe mental illness. Journal of Consulting and Clinical Psychology, 78(4), 561-573. https://doi.org/10.1037/a0019629.

Nowak, I., Switaj, P., Sabariego, C., Oberhauser, C., \& Anczweska, M. (2018). Development and evaluation of a recovery-oriented cognitive behavioural workshop for people diagnosed with schizophrenia. Behaviour and Cognitive Psychotherapy, 47(3), 400-406.

Oertel-Knöchel, V., Mehler, P., Thiel, C., Steinbrecher, K., Malchow, B., Tesky, V., et al. (2014). Effects of aerobic exercise on cognitive performance and individual psychopathology in depressive and schizophrenia patients. European Archives of Psychiatry and Clinical Neuroscience, 264(7), 589-604.

O’Hara, K., Stefancic, A., \& Cabassa, L. J. (2017). Developing a peer-based healthy lifestyle program for people with serious mental illness in supportive housing. Translational Behavioral Medicine, 7(4), 793-803.

Oka, M., Otsuka, K., Yokoyama, N., Mintz, J., Hoshino, K., Niwa, S., et al. (2004). An evaluation of a hybrid occupational therapy and supported employment program in Japan for persons with schizophrenia. American Journal of Occupational Therapy, 58(4), 466-475.

Park, Y. H., \& Han, K. S. (2018). Development and evaluation of a Communication Enhancement Program for People with Chronic Schizophrenia: A quasi-experimental pretest-posttest design study. Applied Nursing Research, 42, 1-8.

Park, S. A., \& Sung, K. M. (2013). The effects on helplessness and recovery of an empowerment program for hospitalized persons with schizophrenia. Perspectives in Psychiatric Care, 49(2), $110-117$.

Pham, M. T., Rajić, A., Greig, J. D., Sargeant, J. M., Papadopoulos, A., \& McEwen, S. A. (2014). A scoping review of scoping reviews: Advancing the approach and enhancing the consistency. Research Synthesis Methods, 5(4), 371-385.

Pioli, R., Vittorielli, M., Gigantesco, A., Rossi, G., Basso, L., Caprioli, C., et al. (2006). Outcome assessment of the VADO approach in psychiatric rehabilitation: A partially randomised multicentric trial. Clinical Practice and Epidemiology in Mental Health, 2(1), 5.

Pratt, S. I., Bartels, S. J., Mueser, K. T., \& Forester, B. (2008). Helping older people experience success (HOPES): An integrated model of psychosocial rehabilitation and health care management for older adults with serious mental illness. American Journal of Psychiatric Rehabilitation, 11, 41-60.

Quee, P. J., Stiekema, A. P. M., Wigman, J. T. W., Schneider, H., van der Meer, L., Maples, N. J., et al. (2014). Improving functional outcomes for schizophrenia patients in the Netherlands using
Cognitive Adaptation Training as a nursing intervention-A pilot study. Schizophrenia Research, 158(1-3), 120-125.

Randal, P., Simpson, A., \& Laidlaw, T. (2003). Can recovery-focused multimodal psychotherapy facilitate symptom and function improvement in people with treatment-resistant psychotic illness? A comparison study. Australian New Zealand Journal of Psychiatry, 37(6), 720-727.

Rapp, C. A., \& Goscha, R. J. (2006). The Strengths Model. A recovery oriented approach to Mental Health Services. Oxford: Oxford University Press.

Resnick, S. G., Fontana, A., Lehman, A. F., \& Rosenheck, R. A. (2005). An empirical conceptualization of the recovery orientation. Schizophrenia Research, 75(1), 119-128.

Ringen, P. A., Falk, R. S., Antonsen, B., Faerden, A., Mamen, A., Rognli, E. B., et al. (2018). Using motivational techniques to reduce cardiometabolic risk factors in long term psychiatric inpatients: A naturalistic interventional study. BMC Psychiatry, 18(1), 255.

Rogers, E. S., Anthony, W. A., Lyass, A., \& Penk, W. E. (2006). A randomized clinical trial of vocational rehabilitation for people with psychiatric disabilities. Rehabilitation Counseling Bulletin, 49(3), 143-156.

Sanches, S. A., Swildens, W. E., van Busschbach, J. T., Stant, A. D., Feenstra, T. L., \& van Weeghel, J. (2015). Cost effectiveness and budgetary impact of the Boston University approach to psychiatric rehabilitation for societal participation in people with severe mental illness: A randomised controlled trial protocol. BMC Psychiatry, 15(1), 217.

Sánchez, P., Peña, J., Bengoetxea, E., Ojeda, N., Elizagárate, E., Ezcurra, J., et al. (2013). Improvements in negative symptoms and functional outcome after a new generation cognitive remediation program: A randomized controlled trial. Schizophrenia Bulletin, 40(3), 707-715.

Sandhu, S., Priebe, S., Leavey, G., Harrison, I., Krotofil, J., McPherson, P., et al. (2017). Intentions and experiences of effective practice in mental health specific supported accommodation services: A qualitative interview study. BMC Health Services Research, 17(1), 471 .

Savla, G. N., Vella, L., Armstrong, C. C., Penn, D. L., \& Twamley, E. W. (2012). Deficits in domains of social cognition in schizophrenia: A meta-analysis of the empirical evidence. Schizophrenia Bulletin, 39(5), 979-992.

Schutt, R. K., Seidman, L. J., Eack, S. M., Deck, B., \& Keshavan, M. S. (2017). Adapting cognitive remediation to a group home: A brief report. Asian Journal of Psychiatry, 25, 184-187.

Scott, D., \& Happell, B. (2011). The high prevalence of poor physical health and unhealthy lifestyle behaviours in individuals with severe mental illness. Issues in Mental Health Nursing, 32(9), 589-597.

Seo, J. M., Ahn, S., Byun, E. K., \& Kim, C. K. (2007). Social skills training as nursing intervention to improve the social skills and self-esteem of inpatients with chronic schizophrenia. Archives of Psychiatric Nursing, 21(6), 317-326.

Sheridan, A., O'Keeffe, D., Coughlan, B., Frazer, K., Drennan, J., \& Kemple, M. (2018). Friendship and money: A qualitative study of service users' experiences of participating in a supported socialisation programme. International Journal of Social Psychiatry, 64(4), 326-334.

Slade, M., Amering, M., Farkas, M., Hamilton, B., O'Hagan, M., Panther, G., et al. (2014). Uses and abuses of recovery: Implementing recovery-oriented practices in mental health systems. World Psychiatry, 13(1), 12-20.

Stiekema, A. P., Quee, P. J., Dethmers, M., van den Heuvel, E. R., Redmeijer, J. E., Rietberg, K., et al. (2015). Effectiveness and cost-effectiveness of cognitive adaptation training as a nursing intervention in long-term residential patients with severe mental 
illness: Study protocol for a randomized controlled trial. Trials, $16(1), 1$.

Stiekema, A. P., Liemburg, E. J., van der Meer, L., Castelein, S., Stewart, R., van Weeghel, J., et al. (2016). Confirmatory factor analysis and differential relationships of the two subdomains of negative symptoms in chronically ill psychotic patients. PLoS ONE, 11(2), e0149785.

Sweeney, R., Moodie, M., Baker, A. L., Borland, R., Castle, D., Segan, C., et al. (2019). Protocol for an economic evaluation of the quitlink randomized controlled trial for accessible smoking cessation support for people with severe mental illness. Frontiers in Psychiatry, 10, 618.

Swildens, W. E., van Busschbach, J. T., Michon, H., Kroon, H., Koeter, M. W., Wiersma, D., et al. (2011). Effectively working on rehabilitation goals: 24-month outcome of a randomized controlled trial of the Boston psychiatric rehabilitation approach. Canadian Journal of Psychiatry, 56(12), 751-760.

Tsang, H. W., \& Pearson, V. (2001). Work-related social skills training for people with schizophrenia in Hong Kong. Schizophrenia Bulletin, 27(1), 133.

Tse, S., Tsoi, E. W., Hamilton, B., O'Hagan, M., Shepherd, G., Slade, M., et al. (2016). Uses of strength-based interventions for people with serious mental illness: A critical review. International Journal of Social Psychiatry, 62(3), 281-291.

van Gestel-Timmermans, J. A. W. M., Brouwers, E. P. M., Bongers, I. L., van Assen, M. A. L. M., \& van Nieuwenhuizen, C. (2012a). Profiles of individually defined recovery of people with major psychiatric problems. International Journal of Social Psychiatry, 58(5), 521-531.

van Gestel-Timmermans, J. A. W. M., Brouwers, E. P. M., van Assen, M. A. L. M., \& van Nieuwenhuizen, C. (2012b). Effects of a peer-run course on recovery from serious mental illness: A randomized controlled trial. Psychiatric Services, 63(1), 54-60.

van Hoof, F., Knispel, A., Aagaard, J., Schneider, J., Beeley, C., Keet, R., et al. (2015). The role of national policies and mental health care systems in the development of community care and community support: An international analysis. Journal of Mental Health, 24(4), 202-207.

van Weeghel, J., van Zelst, C., Boertien, D., \& Hasson-Ohayon, I. (2019a). Conceptualizations, assessments and implications of personal recovery from mental illness: A scoping review of systematic reviews and meta-analyses. Psychiatric Rehabilitation Journal. https://doi.org/10.1037/prj0000356.

van Weeghel, J., van Zelst, C., Boertien, D., \& Hasson-Ohayon, I. (2019). Conceptualizations, assessments, and implications of personal recovery in mental illness: A scoping review of systematic reviews and meta-analyses. Psychiatric Rehabilitation Journal, 42(2), 169-181.

Vandevooren, J., Miller, L., \& O'reilly, R. (2007). Outcomes in community-based residential treatment and rehabilitation for individuals with psychiatric disabilities: A retrospective study. Psychiatric Rehabilitation Journal, 30(3), 215.

Verhaeghe, N., Clays, E., Vereecken, C., de Maeseneer, J., Maes, L., van Heeringen, C., et al. (2013). Health promotion in individuals with mental disorders: A cluster preference randomized controlled trial. BMC Public Health, 13(1), 657.

Wiersma, D. (2006). Needs of people with severe mental illness. Acta Psychiatrica Scandinavica, 113(s429), 115-119.

Willemse, B. M., Depla, M. F., \& Bohlmeijer, E. T. (2009). A creative reminiscence program for older adults with severe mental disorders: Results of a pilot evaluation. Aging \& Mental Health, 13(5), 736-743.

Wong, G., Westhorp, G., Manzano, A., Greenhalgh, J., Jagosh, J., \& Greenhalgh, T. (2016). RAMESES II reporting standards for realist evaluations. BMC Medicine, 14(1), 96.

Publisher's Note Springer Nature remains neutral with regard to jurisdictional claims in published maps and institutional affiliations. 